\title{
AVALIAÇÃO DA DISPONIBILIDADE DE SÓDIO EM MOLHO DE SOJA UTILIZANDO A TÉCNICA DE ESPECTROMETRIA DE ABSORÇÃO ATÔMICA
}

\section{EVALUATION OF THE AVAILABILITY OF SODIUM IN SOY SAUCE USING THE ATOMIC ABSORPTION SPECTROMETRIC TECHNIQUE}

\author{
Vinícius Marques Gomes, Daniel Ângelo Macena, Dayanne Aparecida \\ Dias Pereira, Níckolas Matheus Pereira e Silva, Maíra Rodrigues Uliana \\ Universidade do Oeste Paulista - UNOESTE, Curso de Química Bacharelado, Presidente \\ Prudente, SP. \\ e-mail: daniel@unoeste.br
}

RESUMO - O molho de soja é muito usado em iguarias e seu elevado consumo pode causar hipertensão, uma doença causada pelo consumo de grande quantidade diária de sódio, sendo que o recomendado são dois gramas. Se não tratada, essa doença pode se agravar, gerando problemas cardiovasculares com maior frequência, inclusive levar a óbito. O objetivo deste trabalho foi avaliar o teor de sódio em amostras de Shoyu do tipo Premium, Tradicional e Light, utilizando a técnica de Espectrometria de Absorção Atômica em Chama. Conclui-se que os valores das amostras Light apresentaram maiores concentrações, não condizente com a rotulagem. Esse tipo de molho é muito consumido devido à crença relacionada ao seu baixo teor de sódio, não comprovado com a avaliação realizada. Os valores das versões Tradicionais e Premium foram pertinentes aos da rotulagem.

Palavras-chave: Shoyu; hipertensão; sal; alimento fermentado; espectrometria de absorção atômica.

Recebido em: 06/08/2017

Revisado em: 04/09/2017

Aprovado em: 19/09/2017
ABSTRACT - Soy sauce is widely used in delicacies and its high consumption can cause hypertension, a disease caused by consumption of large daily amount of sodium, and it is recommended to be two grams. If left untreated, this disease can worsen, generating cardiovascular problems more frequently, including leading to death. The objective of this work was to evaluate the sodium content in Shoyu samples of the Premium, Traditional and Light type, using the technique Atomic Absorption Spectrometry in Flame. It is concluded that the values of the Light samples presented higher concentrations, not compatible with the labeling. This type of sauce is very consumed due to the belief related to its low sodium content, not proven in this work. The values of the Traditional and Premium versions are suitable with the labeling.

Keywords: Shoyu; hypertension; salt; fermented food; atomic absorption spectrometry. 


\section{INTRODUÇÃO}

A soja é um alimento em grão considerado funcional, benéfico à saúde por fornecer nutrientes necessários ao homem como: isoflavonas, saponinas, fitatos, inibidores de protease, fitosteróis, peptídeos com baixo peso molecular, oligossacarídeos e ácidos graxos poliinsaturados, que auxiliando na redução de riscos de enfermidades degenerativas e crônicas. Também sendo rica em proteínas e fonte de minerais (CARRÃOPANIZZI et al., 1998).

Este grão pode ser processado e comercializado como uma bebida conhecida como molho de soja (Shoyu), que é um molho fermentado e esta bebida (Shoyu) é amplamente utilizada na gastronomia, principalmente como tempero em iguarias (FUKUSHIMA, 2001).

O molho de soja é uma bebida fermentada originária da China, que antigamente era conhecido como Chian-yu (AQUARONE et al., 2001). Também muito conhecido e consumido nos países ocidentais, sua produção iniciou se no século XIX e foi amplamente estuda pelos franceses e alemães (SHURTLEFF; AOYAGI, 2011).

Após o processo de fabricação realiza-se o envasamento e rotulagem. A rotulagem é fundamental para a segurança alimentar, pois são encontradas as informações necessárias dos produtos industrializados e tais informações são ainda mais relevantes no caso dos alimentos classificados como diet e light (CÂMARA et al., 2008).

Algumas falhas na legislação em questão do seu cumprimento informam valores não correspondentes ao consumidor, principalmente aos que se referem à Informação Nutricional Complementar (INC) e as normas dos alimentos para fins especiais (MARINS; JACOB; TANCREDI, 2005).

Segundo a legislação brasileira (BRASIL, 2001), o consumo de Shoyu não deve ultrapassar uma colher e meia de sopa por dia. Ultrapassar esse valor pode acarretar problemas de saúde, principalmente a hipertensão devido à quantidade de Sódio presente no alimento.

O sódio é um mineral benéfico para a saúde, que atua na manutenção da pressão sanguínea, cerca de $40 \%$ de $\mathrm{NaCl}$ é sódio na sua composição do sal de cozinha. O ideal é consumir $2 \mathrm{~g}$ de sódio por dia, correspondendo a $5 \mathrm{~g}$ de $\mathrm{NaCl}$. Esse consumo, quando controlado, reduz a pressão arterial (PRADO et al., 2016). O consumo excessivo de sódio torna-se um grande problema, levando às pessoas problemas de saúde gravíssimos, entre eles a hipertensão (BANNWART; VIDAL; SILVA, 2014). É recomendado que o consumo de sódio por dia não ultrapasse de 2400 mg (ARAÚJO, 2017). 
Para quantificar o teor de sódio no molho de soja, utilizou-se a técnica de Espectrometria de Absorção Atômica por chama - (FAAS), a qual se baseia na absorção da radiação por átomos livres no estado gasoso (JÚNIOR, 2017).

A FAAS é uma das técnicas mais utilizadas em determinações elementares em níveis traço, pois consiste em atomizar amostras em uma chama e direcionar sobre ela comprimentos de ondas específicos, a qual poderá ser absorvida pelos átomos livres no estado fundamental, desta forma, a redução de energia da radiação no comprimento de onda característico do analítico é uma medida da quantidade desse elemento na amostra (SKOOG et al., 2006).

Diante da problemática e o consumo de Sódio presente no alimentado fermentado, este trabalho teve como objetivo, quantificar o teor de Sódio em diferentes marcas e tipo de molhos de soja e comparar com a rotulagem de cada produto.

\section{METODOLOGIA}

\section{Higienização prévia das vidrarias}

Todas as vidrarias foram higienizadas de acordo com a metodologia proposta por Negrão et al. (2007) utilizando Ácido Nítrico a $30 \%$ v/v em imersão por 2 horas seguido de enxague de 7 vez em água corrente e duas vezes em água destilada. Neste processo foi utilizado tubos digestores de Kjeldahl $(50 \mathrm{~mm}$ $\times 250 \mathrm{~mm} \times 2,5 \mathrm{~mm})$.

O banho em solução de Ácido Nítrico fundamenta-se em descontaminar e/ou eliminar possíveis interferentes na leitura das amostras de forma que reajam com a solução nítrica formando nitratos no qual são solúveis em água.

\section{Seleção das amostras e planejamento do experimento}

As amostras foram adquiridas em supermercados do município de Presidente Prudente-SP. Foram avaliadas três marcas de Shoyu Tradicional, três da versão Light e três da versão Premium. Todos os ensaios analíticos foram realizados em duplicata.

\section{Digestão das amostras por via ácida}

Foram transferidos $50 \mathrm{~mL}$ de cada amostra em diferentes tubos digestores de Kjeldhal e em seguida, adicionados $10 \mathrm{~mL}$ de solução de Ácido Nítrico concentrado e sob aquecimento $(120 \circ \mathrm{C})$ em bloco digestor para tubos macros de 8 provas durante 2 horas, gota a gota foram adicionadas, num total de 20 gotas de peróxido de hidrogênio. Neste processo houve uma mudança de coloração do marrom escuro para alaranjado, onde mostra a dissolução completa da amostra.

Após o período de 2 horas em aquecimento foi deligado o bloco e posteriormente o resfriamento do extrato a temperatura ambiente de forma natural. Em 
seguida, os extratos foram transferidos para balões volumétricos de $100 \mathrm{~mL}$ e o volume dos balões completados com água destilada totalizando no fim dezoito amostras para serem posteriormente analisadas.

\section{Quantificação de Sódio nas amostras de Shoyu}

A técnica para determinação do teor total de sódio no molho de soja foi realizada no aparelho espectrometria de Absorção Atômica em Chama (FASS), utilizando o comprimento de onda de $589 \mathrm{~nm}$. O equipamento utilizado foi o modelo AAnalyst 200 de marca Perkin Elmer.

\section{RESULTADOS}

Tabela 1. Dados experimentais e dados dos rótulos de cada marca de Shoyu.

\section{Tipos de Molho}

\begin{tabular}{crrrrcr} 
Marcas & \multicolumn{2}{c}{ Tradicional $(\mathrm{mg} / \mathrm{mL})$} & \multicolumn{2}{c}{ Premium $(\mathrm{mg} / \mathrm{mL})$} & \multicolumn{2}{c}{ Light $(\mathrm{mg} / \mathrm{mL})$} \\
& MA & $R$ & MA & R & MA & R \\
\hline $\mathbf{1}$ & 493 & 501 & 421 & 540 & 420 & 342 \\
\hline $\mathbf{3}$ & 674 & 818 & 660 & 818 & 708 & 570 \\
\hline & 271 & 795 & 257 & 770 & 532 & 441 \\
\hline
\end{tabular}

Fonte: Próprio autor

Nota: $\mathrm{MA}=$ Média das Amostras; $\mathrm{R}=$ Dados disponíveis nos rótulos de cada produto.

Gráfico 1. Análise geral das concentrações de Sódio disponível nos diferentes molhos de soja a partir da média amostral e comparação a rotulagem do produto nos tipos Tradicional, Premium e Light.

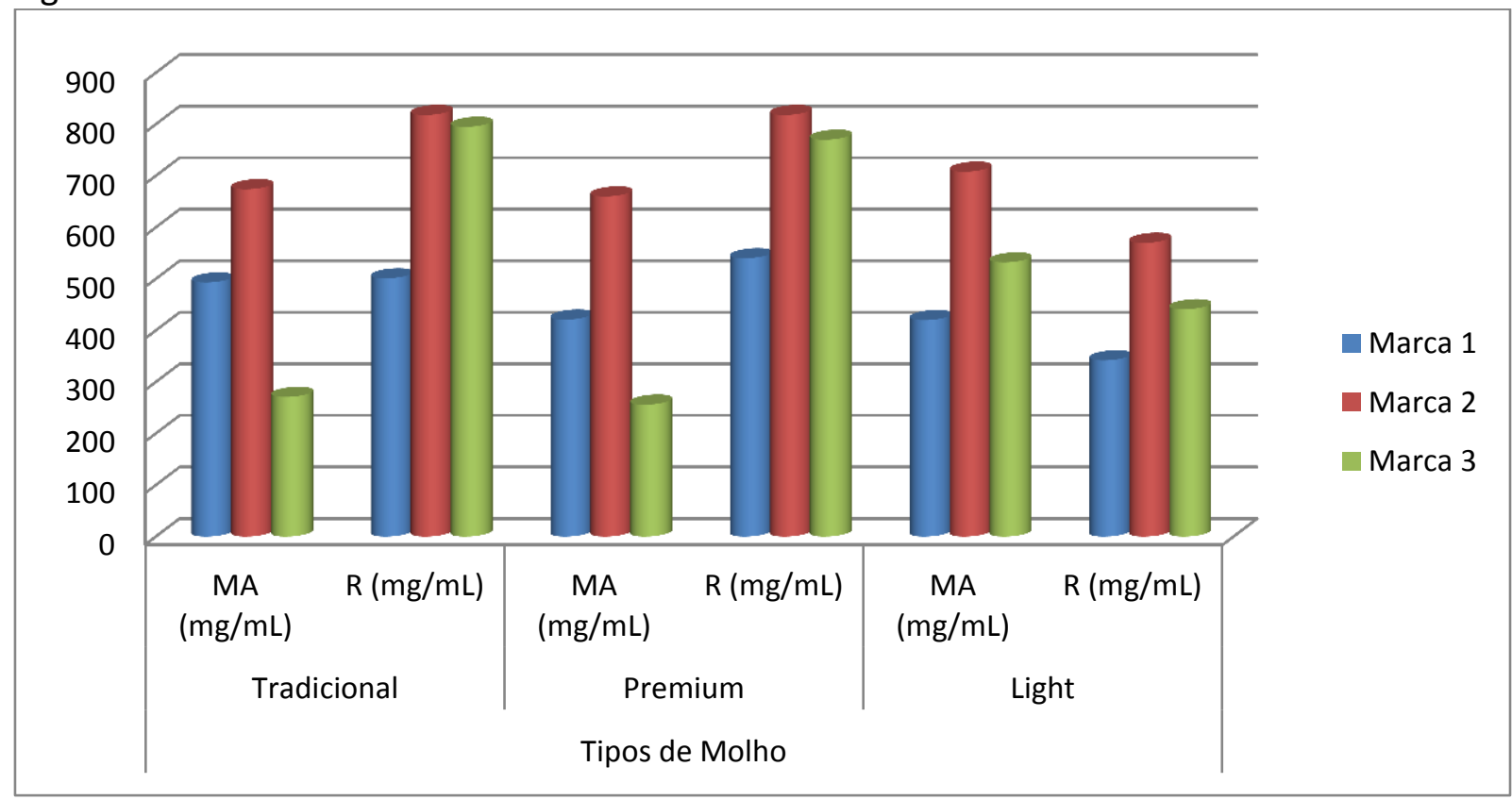

Fonte: Próprio autor

Nota: $\mathrm{MA}=$ Média das Amostras; $\mathrm{R}=$ Dados disponíveis nos rótulos de cada produto. 
Conforme a tabela 1 seguem as médias dos resultados amostrais e os dados fornecidos pelos respectivos fabricantes das três marcas diferentes de molho de soja (Shoyu) que foram denominadas marca 1, 2 e 3 para assegurar a imagem dos fabricantes.

Segue a representação gráfica (Gráfico 1), histograma, em barras verticais da distribuição de frequências do conjuntos de resultados quantitativos para a compreensão e comparação dos valores, entre os dados experimentais e os fornecidos na rotulagem dos molhos pelos fabricantes correspondentes conforme descritos na tabela 1.

\section{DISCUSSÃO}

Ao observarmos o gráfico 1 percebese que dentre as três marcas diferentes apenas as versões lights apresentaram acima do que foi especificado nos rótulos originais dos produtos, podendo concluir que as versões lights estão incoerentes com a afirmação de que os produtos lights desta marca, ao menos neste lote apresentam menores teor de Sódio no alimento. De acordo com os resultados analíticos ainda para o tipo light de molho de soja as marcas 1, 2 e 3 apresentaram $78 \mathrm{mg} / \mathrm{mL}, 138 \mathrm{mg} / \mathrm{mL}$ e $91 \mathrm{mg} / \mathrm{mL}$, respectivamente, acima do que especificado no rótulo original.

Percebe-se também no gráfico 1 que relacionando as três diferentes marcas com a tabela nutricional disponibilizada pelo fabricante é possível estabelecer uma ordem de quantidade de Sódio no produto, pois a marca 2 em todos os resultados é a que apresenta a maior quantidade de Sódio seja no molho tradicional, premium ou light. Em segundo lugar a marca 3 representa a maior quantidade, ainda que nos resultados analíticos tenha apresentado valores abaixo, no tipo tradicional e premium, do que foi estabelecido na tabela nutricional. Por fim, a marca 1 neste estudo, em relação ao lote utilizado como amostra, foi a marca que mais aproximou-se dos valores referentes a tabela nutricional original do produto, exceto o molho light que apresentou-se acima do indicado. Em ralação a ordem de disponibilidade de Sódio nos molhos é importante para se certificar quanto a qualidade dos produtos que estão sendo ofertados nos mercados.

De acordo com um estudo realizado no Japão pela Hamamatsu University School of Medicine, a substituição do molho de soja na versão tradicional pela versão light não ocasionou alterações significativas na pressão sanguínea do grupo avaliado, exceto pessoas que possuíam 40 anos ou mais, onde a pressão diastólica foi reduzida em $6 \mathrm{mmHg}$. Os autores afirmam que o molho de soja baixo em sódio possui entre $20 \%$ a $25 \%$ menos sódio. Foram avaliados 40 homens e 24 mulheres, divididos em grupo de baixo sódio e grupo controle sem levar em 
consideração diferenças de idade, sexo, massa corporal, pressão sanguínea ou hipertensão entre os dois grupos antes da intervenção. Concluíram que a substituição do molho tradicional pela versão light é uma boa estratégia na dieta da população como estratégia para redução de sal na dieta japonesa (NAKAMURA et al., 2003). Relacionando ao presente estudo fica como sugestão estudos complementares ou até mesmo um monitoramento em diferentes lotes das marcas em estudo já que todas apresentaram-se fora do padrão especificado nos rótulos.

Outro estudo por Spinelli, Kawashima e Egashira (2011) diz que apesar de os restaurantes self-service ser uma alternativa para refeições fora de casa, existe uma preocupação com combinação e sabores, porém a adequação nutricional não é o objetivo principal. Portanto este foi o motivo para que analisassem o teor de sódio disponível em três alimentos (arroz, feijão e carne) consumidos de 15 diferentes restaurantes na cidade de Mogi das CruzesSP através da técnica de fotometria de chama por emissão atômica. Todas as análises procederam-se a partir de $100 \mathrm{~g}$ do alimento específico e como resultado desta pesquisa todas as refeições apresentaram-se também concentrações altas de Sódio ao qual foi sugerido a necessidade de estabelecer padrões em restaurantes comerciais. Em relação às recomendações, apenas com os três alimentos em estudo, todas as porções excederam em $25 \%$ de Sódio sugerida pelo guia alimentar em uma única refeição. Percebe-se neste estudo que o Sódio em geral é uma preocupação por estarem presentes na dieta diária das pessoas, porém a preocupação quanto a dosagem e qualidade nutricional é um problema que precisa ser sanado tanto quando pensa-se no produto industrializado no caso do presente estudo ou quando pensamos no produto preparado na hora como nos restaurantes.

A RDC no 360/2003 é o Regulamento Técnico sobre Rotulagem Nutricional de Alimentos Embalados na qual fornece as diretrizes sobre a rotulagem específica nutricional obrigatória com declarações de informações a respeito de valores energéticos, carboidratos, proteínas, gorduras totais, gorduras saturadas, gorduras trans, fibra alimentar e sódio. Portanto existe a ANVISA (2003) que dispõe os requisitos básicos aos fabricantes quanto a qualidade e informações nutricionais dos produtos que são oferecidos comercialmente.

\section{CONCLUSÃO}

A possibilidade de consultar o rótulo do produto na hora da compra representa um papel muito importante referente à educação nutricional, é através desta leitura que o consumidor escolhe com eficiência o 
produto adequado à sua realidade. Para isso, as informações presentes no rótulo devem estar corretas.

Conclui-se neste estudo que é necessário uma fiscalização mais rigorosa em relação ao alimento, pois somente a leitura dos rótulos não garante a dieta nutricional ao que é esperado pelo consumidor, além do que o alimento em estudo apresenta uma importância significativa dentro da culinária na combinação de pratos e sabores.

\section{REFERÊNCIAS}

AQUARONE, E. et al. Biotecnologia industrial: biotecnologia na produção de alimentos. Edgard Blucher, 2001.

ANVISA. Resolução da Diretoria Colegiada no. 360, de 23 de dezembro de 2003. Disponível em:

<http://www.anvisa.gov.br/legis/resol/2003/ rdc/360_03rdc.htm>. Acesso em: 03 ago. 2017.

BANNWART, G. C. M. C.; VIDAL, G.; SILVA, M. E. M. P. Redução de sódio em alimentos: panorama atual e impactos tecnológicos, sensoriais e de saúde pública. Nutrire Rev. Soc. Bras. Aliment. Nutr, v. 39, n. 3, 2014.

BRASIL. Ministério da Agricultura Pecuária e Abastecimento. 2001. Disponível em: <http://sistemasweb.agricultura.gov.br>. Acesso em: 03 ago 2017.

CÂMARA, M. C. C. et al. A produção acadêmica sobre a rotulagem de alimentos no Brasil. Rev Panam Salud Publica, v. 23, n. 1, p. 52-8, 2008. https://doi.org/10.1590/S102049892008000100007

CARRÃO-PANIZZI, C. M. et al. Influence of growth locations on isoflavone contents in
Brazilian soybean cultivars. Japanese Journal of Breeding, v. 48, n. 4, p. 409-413, 1998. https://doi.org/10.1270/jsbbs1951.48.409

FUKUSHIMA, D. Recent Progress in Research and Technology on Soybeans. Food Science and Technology Research, v. 7, n. 1, p. 8-16, 2001. https://doi.org/10.3136/fstr.7.8

JÚNIOR, Q. et al. Utilização da membrana de quitosana como alternativa para tratamento de efluentes com metais pesados. 2017. Trabalho (Conclusão de Curso) - Universidade Federal do Rio Grande do Norte, 2017.

MARINS, B. R.; JACOB, S. C.; TANCREDTI, R. C. $P$. A rotulagem de alimentos praticada pelo estabelecimento fracionador. Será que obedece a legislação vigente?. Hig. Aliment., p. 121-126, 2005.

NAKAMURA, $M$. et al. Feasibility and effect on blood pressure of 6-week trial of low sodium soy sauce and miso (fermented soybean paste). Circulation Journal, v. 67, n. 6, p. 530-534, 2003. https://doi.org/10.1253/circj.67.530

NEGRÃO, A. N. et al. Validação de métodos de lavagem de vidraria utilizando vitamina B12 como marcador de limpeza. Latin American Journal of Pharmacy, v. 26, n. 2, p. 280, 2007.

PRADO, B. G. et al. Ações de educação alimentar e nutricional para escolares: um relato de experiência. DEMETRA: Alimentação, Nutrição \& Saúde, v. 11, n. 2, p. 369-382, 2016.

SHURTLEFF, W.; AOYAGI, A. History of soy sprouts. A special report on the history of traditional non-fermented soya foods. A Chapter from the Unpublished Manuscript, History of Soybeans and Soyfoods: 1100 BC to the 1980s. Soyinfo Center, v. 1, p. 2011, 2011. 
SKOOG, D. A. et al. Fundamentos de química analítica. 8. ed. Thomson, 2006.

SPINELLI, M. G. N.; KAWASHIMA, L. M.; EGASHIRA, E. M. Análise de sódio em preparações habitualmente consumidas em restaurantes self service. Brazilian Journal of Food \& Nutrition/Alimentos e Nutrição, v. 22, n. 1, 2011. 